\title{
Long Bone X-Ray Image Stitching Using Camera Augmented Mobile C-Arm
}

\author{
Lejing Wang ${ }^{1}$, Joerg Traub ${ }^{1}$, Sandro Michael Heining ${ }^{2}$, Selim Benhimane ${ }^{1}$, \\ Ekkehard Euler ${ }^{2}$, Rainer Graumann ${ }^{3}$, and Nassir Navab ${ }^{1}$ \\ ${ }^{1}$ Chair for Computer Aided Medical Procedures (CAMP), TU Munich, Germany \\ 2 Trauma Surgery Department, Klinikum Innenstadt, LMU Munich, Germany \\ ${ }^{3}$ Siemens SP, Siemens Medical Solutions, Erlangen, Germany
}

\begin{abstract}
X-ray images are widely used during surgery for long bone fracture fixation. Mobile C-arms provide X-ray images which are used to determine the quality of trauma reduction, i.e. the extremity length and mechanical axis of long bones. Standard X-ray images have a narrow field of view and can not visualize the entire long bone on a single image. In this paper, we propose a novel method to generate panoramic X-ray images in real time by using the previously introduced Camera Augmented Mobile C-arm [1. This advanced mobile C-arm system acquires registered X-ray and optical images by construction, which facilitates the generation of panoramic X-ray images based on first stitching the optical images and then embedding the X-ray images. We additionally introduce a method to reduce the parallax effect that leads to the blurring and measurement error on panoramic X-ray images. Visual marker tracking is employed to automatically stitch the sequence of video images and to rectify images. Our proposed method is suitable for intra-operative usage generating panoramic X-ray images, which enable metric measurements, with less radiation and without requirement of fronto-parallel setup and overlapping X-ray images. The results show that the panoramic X-ray images generated by our method are accurate enough (errors less than $1 \%$ ) for metric measurements and suitable for many clinical applications in trauma reduction.
\end{abstract}

\section{Introduction}

X-ray images are the most frequently used form of medical imaging during orthopaedic surgery and provide good contrast of bone structures. Mobile C-arms are an everyday tool to acquire X-ray images in the operating room during surgery. Currently, long bone fracture fixation heavily relies on intraoperative $\mathrm{X}$-ray images. The limited field of view is a drawback of X-ray mobile C-arm imaging. In long bone fracture fixation surgery, surgeons need to measure the length of the bone to be reconstructed and align the bone fragments. Single $\mathrm{X}$-ray image can not visualize the entire long bone. Surgeons are required to acquire several individual X-ray images and correlate them.

Panoramic X-ray images can be obtained by stitching many X-ray images. A special purpose digital X-ray machine was introduced to generate panoramic

D. Metaxas et al. (Eds.): MICCAI 2008, Part II, LNCS 5242, pp. 578-586, 2008.

(C) Springer-Verlag Berlin Heidelberg 2008 
X-ray images by simultaneously translating the X-ray source and the image intensifier over the patient 2]. This X-ray machine is not versatile enough to replace mobile $\mathrm{C}$-arms within operating rooms. Therefore an intra-operative solution based on mobile C-arms is still required. Currently, the correct fracture reduction can only be confirmed post-operatively. For obtaining an X-ray panoramic image intra-operatively two methods were proposed using standard mobile $\mathrm{C}$-arms. The first system introduces an orthopaedic radiolucent $\mathrm{X}$-ray ruler placed along the bones [3]. It uses the graduations of the ruler on the $\mathrm{X}$ ray images to estimate the planar transformation by a feature-based alignment method and requires the user to manually select the reconstruction plane in order to compensate for parallax effects on that plane. Another method employs a radio-opaque absolute reference panel with absolute coordinates placed under the bones 4. This reference panel contains a grid of radio-opaque rigid markers and thus X-ray images can be registered based on the known geometry of this panel. However, both methods have their limitations. The first method 3 requires overlapping areas between two consecutive X-ray images to estimate the planar transformation and thus requires additional radiation, while the second method 4 is independent from overlapping X-ray regions, but requires an X-ray visible panel and does not show how to solve for the parallax effects, that are introduced if the stitching plane and the target plane are not the same. Both methods require a frontal parallel C-arm setup, i.e. the stitching plane must be parallel to the detector of $\mathrm{C}$-arms.

The Camera Augmented Mobile C-arm (CAMC) system that extends a regular mobile C-arm by a video camera [1] was proposed for X-ray and video image overlay. By construction of the device, the acquired X-ray images are co-registered with the video images. Our work makes the CAMC system capable of generating panoramic X-ray images intra-operatively without overlapping X-ray images and special X-ray markers. Using the video images for stitching and finally showing their co-registered X-ray images facilitates the creations of panoramic X-ray images. The major advantages over previous proposed solutions are the reduction of ionizing radiation, not requiring frontal parallel $\mathrm{C}$-arm setup, and enabling metric measurements. This results in new applications and enables the confirmation of the trauma reduction within the surgery room.

\section{System Components}

\subsection{System Setup}

Our Camera Augmented Mobile C-arm system is composed of a mobile C-arm, Siremobile Iso-C 3D, from Siemens Medical Solutions and an optical video camera, Flea, from Point Grey Research Inc.. The camera is connected via firewire connection (IEEE-1394) to the computer with custom developed software. The computer is a standard PC extended by a frame grabber card, Falcon, from IDS Imaging Development System GmbH. Thanks to a custom made attachment of the camera and a double mirror construction, the camera center virtually coincides 
with the X-ray source. To register X-ray images to optical images, the proposed offline calibration of [1] is used.

\subsection{Method}

Image stitching has been intensively studied in the last decades [5] in non medical areas. One central component of stitching is image registration, i.e. the estimation of a transformation to align two images. Intensity-based registration needs large overlap between the consecutive X-ray images, which is undesirable for $\mathrm{X}$-ray image stitching since it increases the radiation. On the other hand, it is hard to detect anatomical features reliably and accurately in the X-ray images for feature-based registration. This is one reason that a radiolucent X-ray ruler [3] and a radio-opaque absolute reference panel [4] were introduced.

In our setup the X-ray images are registered with their corresponding video images by construction of the Camera Augmented Mobile C-arm system. The video provides a series of images with features, in which two consecutive images have large overlap. We first stitch the sequence of video images and then overlay the registered X-ray images onto their corresponding video images (see figure 1). Finally, we are able to generate a panoramic video image, and using the co-registered X-ray images, we create the panoramic X-ray image.

All video images are acquired sequentially. A simple pattern is used to stitch the image sequence. The first acquired image is defined as the reference image, and all others will be registered into coordinate system of this first image (see figure 11). ${ }^{i-1} H_{i}^{v} \in \mathbb{R}^{3 \times 3}$ denotes the planar homography used to register each

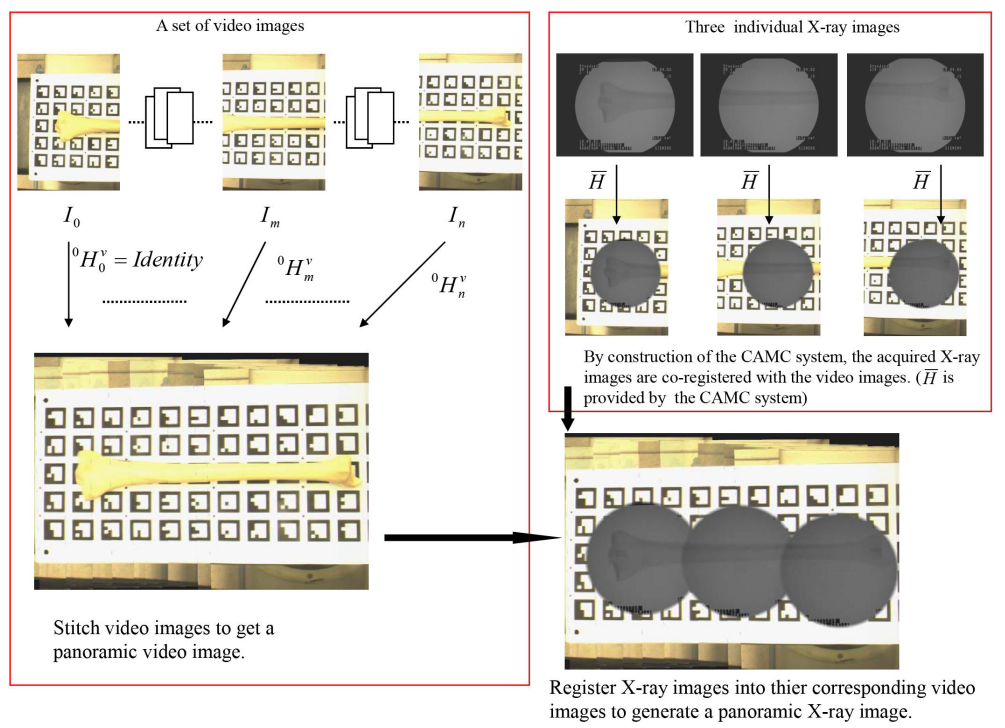

Fig. 1. The idea of X-ray image stitching using the camera augmented mobile C-arm system and a square marker pattern 
image $I_{i}$ to its predecessor $I_{i-1}$, and can be estimated by registering two consecutive nearly identical video images. The image $I_{i}$ can be registered to the image $I_{0}$ by homography ${ }^{0} H_{i}^{v}={ }^{0} H_{1}^{v} \cdot{ }^{1} H_{2}^{v} \ldots{ }^{i-2} H_{i-1}^{v} \cdot{ }^{i-1} H_{i}^{v}$.

After stitching a video sequence, the X-ray images will be overlaid onto their corresponding video images by the homography $\bar{H}$ and thus they are also registered within the coordinate system of the first video image (see figure 1). This $\bar{H}$ is provided by the CAMC system and is calculated during the offline calibration when the CAMC is manufactured [1]. Based on this derivation, we can enable the CAMC system to generate panoramic X-ray images intra-operatively by stitching the acquired video images. All homographies for stitching video images and registering X-ray images are estimated independently from acquired X-ray images. Thus, two non-overlapping X-ray images can be stitched.

\subsection{Parallax Effect}

The planar homography that aligns two consecutive video images is represented by ${ }^{i-1} H_{i}^{v}$. In [3] this planar homography is defined by ${ }^{i-1} H_{i}^{v}=K R K^{-1}+$ $\frac{1}{d} K t n^{T} K^{-1}$ whose $K \in \mathbb{R}^{3 \times 3}$ is the intrinsic matrix of the camera, $R \in \mathbb{R}^{3 \times 3}$ the rotation and $t \in \mathbb{R}^{3}$ the translation of the camera motion. ${ }^{i-1} H_{i}^{v}$ is valid for all image points whose corresponding space points are on the same plane, called stitching plane, defined by the normal vector $n \in \mathbb{R}^{3}$ and distance $d$ to the origin in the world coordinate system. However, any structure that is not within this stitching plane in 3D space will get ghosting or blurring effects called parallax. Since the ${ }^{i-1} H_{i}^{v}$ is estimated by video images, there are always parallax effects on the bone reconstruction plane. The parallax effect will cause not only the blurring, but also the measurement errors on the image.

In this work, a method is proposed to reduce parallax effects. Our method relies on three assumptions: 1) images are obtained from a frontal parallel view of the stitching plane (we succeeded however in removing this constriant, see section 2.4);2) translating the $\mathrm{C}$-arm parallel to the stitching plane during the image acquisition; 3) the stitching plane is parallel to the bone plane used for panoramic X-ray images and metric measurements. Based on these assumptions, $R$ is an identity matrix, and we have the homography ${ }^{i-1} H_{i}^{x}$ for the bone plane ${ }^{i-1} H_{i}^{x}=K R K^{-1}+\frac{1}{d+\Delta d} K t n^{T} K^{-1}=I+\frac{d}{d+\Delta d}\left(\frac{1}{d} K t n^{T} K^{-1}\right)$. Both $^{i-1} H_{i}^{v}$ and ${ }^{i-1} H_{i}^{x}$ are $2 \mathrm{D}$ translation mappings. Eventually, the unknown translation part $\left(x_{t}^{x}, y_{t}^{x}\right)$ of ${ }^{i-1} H_{i}^{x}$ can be computed by $x_{t}^{x}=s x_{t}^{v}$ and $y_{t}^{x}=s y_{t}^{v}$, in which $\left(x_{t}^{v}, y_{t}^{v}\right)$ is the known translation part of ${ }^{i-1} H_{i}^{v}$, the homography on the stitching plane, and $s=\frac{d}{d+\Delta d}$, the distance ratio. So far, all variables are known except $s$.

Since all images are acquired from a frontal parallel view of the stitching plane, the distance ratios and angles of the stitching plane are preserved on the image. We estimate the distance $d$ from the stitching plane to camera center by constructing a known distance on that plane and measuring its image distance with a known camera model. To estimate the homography ${ }^{i-1} H_{i}^{x}$, we need to estimate $\Delta d$, the distance between the stitching plane and the bone plane. In the intramedullary tibial fracture reduction surgery, there are two planes: the tibial plane and the nail plane. Surgeons need to define the $\Delta d$, depending 
on their preferences of the plane to be visualized and used to perform metric measurements.

With this derivation, it is possible to estimate the homography ${ }^{i-1} H_{i}^{x}$ directly from the known ${ }^{i-1} H_{i}^{v}$ without explicitly computing the translation $t$. This method depends on the accuracy of the estimated $\Delta d$ by surgeons. Table 2 shows the results of an experiment for evaluating the influence of perturbed $\Delta d$, denoted as $\Delta d^{\prime}$, on metric measurement accuracy on the final panoramic X-ray images.

\subsection{Frontal Parallel Setup and Implementation Details}

The frontal parallel setup (i.e. stitching plane parallel to the image plane) is the prerequisite to perform metric measurements. However, this is not easy to establish and adds an additional challenge for surgeons to achieve an optimal initial setup. By using an optical pattern we can rectify X-ray images onto the stitching plane to preserve metric properties. Finally our method only relies on two assumptions: 1) the X-ray is moving parallel to the stitching plane with no restriction on the orientation of the image intensifier. This is in contrast to the traditional methods (e.g. Ziv et al.), which require both x-ray source motion and the image intensifier orientation to be parallel to the bony structure; 2) the stitching plane is parallel to the bone plane used for panoramic X-ray images and metric measurements.

A marker tracking system is able to detect markers fully automatically 6. In our implementation, all square markers are positioned on the same plane, the stitching plane. Since these coplanar markers provide sufficient corresponding points on the stitching plane, the Normalized Direct Linear Transformation (DLT) 7] can be used to estimate the homography ${ }^{i-1} H_{i}^{v}$. Fixing the relative positions of square markers with known size, we are able to establish point correspondences between the points in the image plane and in the stitching plane. Based on these point correspondences, a homography is estimated to rectify images onto the stitching plane. We designed a pattern (see figure 2(a) with multiple square markers that can be uniquely identified.

\section{Experiments and Results}

The phantom (see figure 2) used throughout all our experiments is constructed to have two parallel planes with an adjustable distance $\Delta d$. On the upper plane, the marker plane for stitching, the marker pattern is attached. On the lower plane, the bone plane, we placed X-ray markers with known distances for metric measurements or a bone phantom for feasibility tests.

The image distance is defined by clicking two points (three points for an angle). To determine the accuracy of metric measurements, we used spherical X-ray markers instead of a bone, since we can extract the center of a circle with subpixel accuracy. The ground truth of metric measurements is built by attaching X-ray markers on a pattern with known metric properties. The accuracy of the 


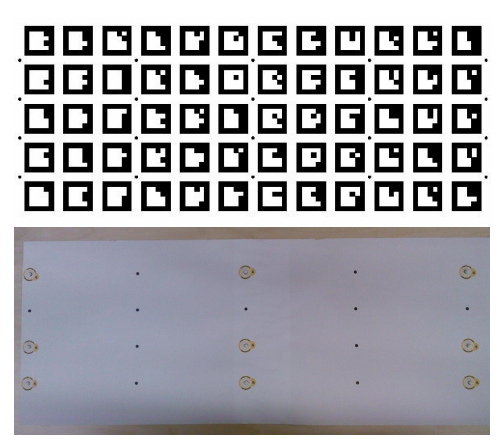

(a) The marker pattern (top) and X-ray markers (bottom).
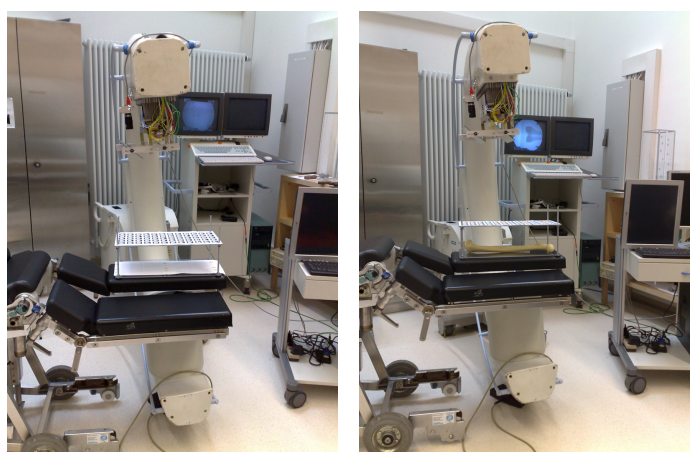

(c) The system setup for feasibility tests.

Fig. 2. Our designed phantom for experiments. The marker pattern is attached on the upper plane. The X-ray marker and the bone are placed on the lower plane.

attachments was controlled by a ruler, thus the error in the ground truth was confirmed to be below $1 \mathrm{~mm}$. Our phantom with the X-ray marker was placed on the operating table (see figure 2(b) such that the stitching plane deviates several degrees from the image intensifier to simulate the general cases. While acquiring video and x-ray images, the operating table was moved through the $\mathrm{C}$-arm and the panoramic image was created. We measured several different distances or angles with the ground truth value of $480 \mathrm{~mm}$ or 161.0754 degree respectively, on the same image to evaluate image distortion and eliminate bias error in the results. Table 1 shows the distance and angle measurement results. Without applying depth transformation, both the absolute and relative errors will increase as $\Delta d$ increases. After applying our depth compensation method (see section 2.3), the absolute errors are similar to the one in the video panoramic images, and the relative errors are about constant. The small standard deviation indicates that our panoramic images have minor distortion.

We also evaluated the influence of errors in estimating $\Delta d$ on the accuracy of metric measurements. Instead of $\Delta d=100 \mathrm{~mm}$, we take $\Delta d^{\prime}$ varying between $0 \mathrm{~mm}$ and $170 \mathrm{~mm}$ to get panoramic X-ray images that enable metric measurements (see table 2). The relative error will increase with the increase of the difference between the true depth and the assumed one $\left(\Delta d^{\prime}-\Delta d\right)$. One expects to get the minimal relative error at $\Delta d=100 \mathrm{~mm}$. However, we obtained minimal relative error of $0.2 \mathrm{~mm}$ at $\Delta d^{\prime}=110 \mathrm{~mm}$. The overlay error of X-ray image and video image introduced in the offline calibration of our CAMC system also contributes to that relative error (the mean error of the overlay was measured to be $0.5 \mathrm{~mm}$ ). The experiment shows furthermore that a deviation of $10 \mathrm{~mm}$ in the depth estimation causes to less than $1 \mathrm{~mm}$ error over $480 \mathrm{~mm}$ true distance in the metric measurements. 
Table 1. Actual and measured distances/angles on the panoramic images in $\mathrm{mm} /$ degrees. The absolute error (mean \pm std) is between the ground truth and the extracted value. The relative error is between the video stitching and the $\mathrm{X}$-ray stitching.

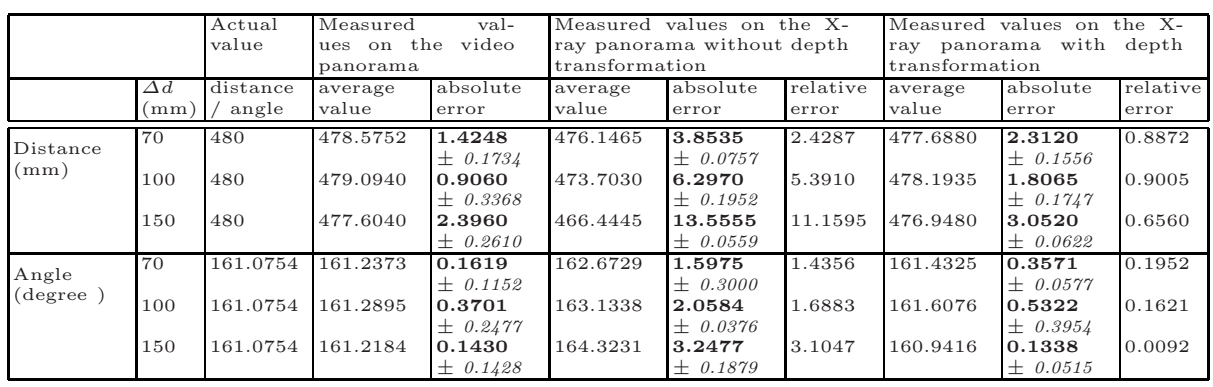

Table 2. Measured distances on panoramic X-ray images after applying depth transformation by using perturbed depth

\begin{tabular}{|c|c|c|c|c|c|c|c|c|c|c|}
\hline$\Delta d^{\prime}(\mathrm{mm})(\Delta d=100 \mathrm{~mm})$ & 0 & 30 & 70 & 80 & 90 & 100 & 110 & 120 & 130 & 170 \\
\hline Relative error $(\mathrm{mm})$ & 5.2 & 3.8 & 2.0 & 1.6 & 1.1 & 0.6 & 0.2 & 0.3 & 0.7 & 2.6 \\
\hline $\begin{array}{lll}\text { Measured distance on the video } \\
\text { panorama (mean value) }(\mathrm{mm})\end{array}$ & 479.1 & & & & & & & & & \\
\hline Actual distance $(\mathrm{mm})$ & 480 & & & & & & & & & \\
\hline
\end{tabular}

\section{Discussion and Conclusion}

Panoramic X-ray images are a promising technology for determining the extremity length and mechanical axis of long bones online during surgeries. In our work, we presented a new method to generate panoramic X-ray images intra-operatively by using the Camera Augmented Mobile C-arm system and a planar maker pattern. The square marker pattern is only needed during generation of panoramic X-ray images and removed from complete the surgical task. In any orthopedic surgery the intraoperative X-ray acquisition always interrupts the surgical procedure for a short while during which the staff tries to stay away from direct radiation. The proposed solution integrates smoothly into the surgical procedure as the pattern is only used during the same window of time. In order to investigate the clinical value, we performed preclinical experiments and generated panoramic X-ray images of a bone phantom (see figure 2(c). The panoramic X-ray image was generated from four individual X-ray images. Figure 3 shows the panoramic images. Our method is independent from overlapping $\mathrm{X}$-ray regions and does not require a frontal parallel C-arm setup. The experimental results show that the panoramic $\mathrm{X}$-ray images generated by our method have a high visual quality and are accurate enough for metric measurements. The absolute errors were less than $1 \%$ and relative errors were even below $0.5 \%$.

One of the major challenges within X-ray stitching is the parallax effect. We compensate for it by our depth transformation whose accuracy depends on the depth difference between the stitching plane and the bone reconstruction plane. 

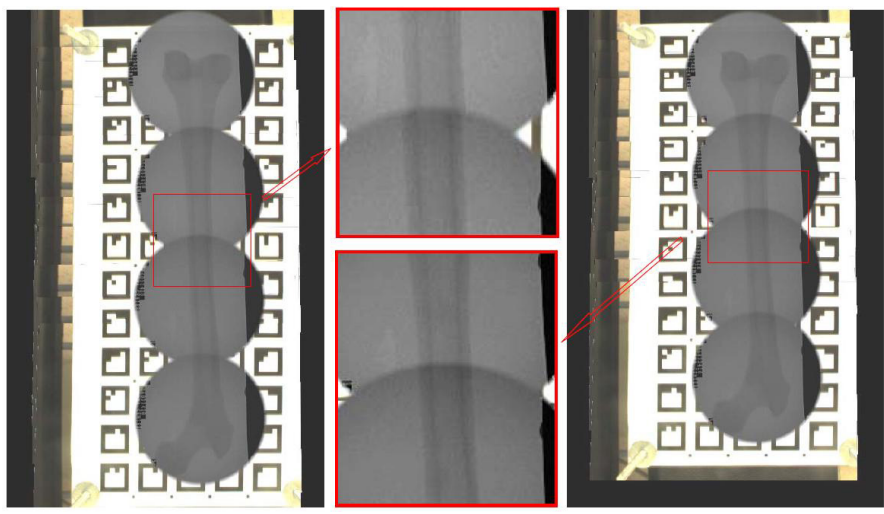

Fig. 3. The X-ray panoramic image generated by our method. The left picture is without depth transformation and discontinuous (middle top shows) appear at the overlapping areas. The right picture shows the X-ray panoramic after applying depth transformation. This compensated for the discontinuous (middle bottom shows).

In general, this distance is not trivial to recover. In our preclinical phantom setup we can directly measure the distance $\Delta d$. Appropriate methods have to be incorporated to define the bone plane and estimate the depth transformation. Clinical tests in the near future will show the feasibility and the advantage of metric distance and angle measurements online within the surgery room based on a standard C-arm. This will allow surgeons to ensure and validate during trauma reduction surgery the quality of their treatment.

Acknowledgment. Thank to Siemens Medical Solutions for partial financial support.

\section{References}

1. Navab, N., Mitschke, M., Bani-Hashemi, A.: Merging visible and invisible: Two camera-augmented mobile C-arm (CAMC) applications. In: Proc. IEEE and ACM Int'l Workshop on Augmented Reality, San Francisco, CA, USA, pp. 134-141 (1999)

2. Geijer, H., Beckman, K.W., Jonsson, B., Andersson, T., Persliden, J.: Digital radiography of scoliosis with a scanning method: Initial evaluation. Radiology 218(2), 402-410 (2001)

3. Yaniv, Z., Joskowicz, L.: Long bone panoramas from fluoroscopic x-ray images. IEEE transactions on medical imaging 23(1), 26-35 (2004)

4. Messmer, P., Matthews, F., Wullschleger, C., Hgli, R., Regazzoni, P., Jacob, A.L.: Image fusion for intraoperative control of axis in long bone fracture treatment. European Journal of Trauma 32, 555-561 (2006)

5. Szeliski, R.: Image alignment and stitching: A tutorial. Technical report, Microsoft Research (2006) 
6. Zhang, X., Fronz, S., Navab, N.: Visual marker detection and decoding in ar systems: A comparative study. In: IEEE International Symposium on Mixed and Augmented Reality (ISMAR 2002) (October 2002)

7. Hartley, R., Zisserman, A.: Multiple View Geometry in Computer Vision. Cambridge University Press, New York (2003) 